PROCEEDINGS OF THE

AMERICAN MATHEMATICAL SOCIETY

Volume 130, Number 5, Pages 1383-1389

S 0002-9939(01)06353-5

Article electronically published on October 17, 2001

\title{
LOCAL BOUNDARY DILATATION OF QUASICONFORMAL MAPS IN THE DISK
}

\author{
GUIZHEN CUI AND YI QI
}

(Communicated by Juha Heinonen)

\begin{abstract}
In this paper we partly give an affirmative answer to a problem proposed by F. Gardiner and N. Lakic by studying the gluing of quasiconformal maps.
\end{abstract}

\section{INTRODUCTION}

Let $\Omega$ be a plane domain with at least two boundary points and let $M(\Omega)$ be the open unit ball of $L^{\infty}(\Omega)$. Every element $\mu \in M(\Omega)$ can be regarded as an element in $L^{\infty}(\mathbf{C})$ by putting $\mu$ equal to zero in the outside of $\Omega$, where $\mathbf{C}$ is the complex plane. For any such $\mu$ there is a quasiconformal (q.c., for brevity) self-map $f^{\mu}$ of the complex plane $\mathbf{C}$ that solves the Beltrami equation

$$
f_{\bar{z}}(z)=\mu(z) f_{z}(z)
$$

and $f^{\mu}(z)$ is determined uniquely up to postcomposition by a complex affine map of the plane. The solution $f^{\mu}$ is said to be induced by $\mu$. Conversely, for every quasiconformal map $f$ defined in $\Omega$ there is a measurable function $\mu_{f}=f_{\bar{z}}(z) / f_{z}(z) \in$ $M(\Omega)$ which is called the complex dilatation or the Beltrami coefficient of $f$. The constant

$$
K(f)=\frac{1+\|\mu\|_{\infty}}{1-\|\mu\|_{\infty}}
$$

is called the maximal dilatation of $f$.

The Teichmüller space $T(\Omega)$ of $\Omega$ is the space of equivalence classes of Beltrami coefficients in $M(\Omega)$. Two Beltrami coefficients $\mu$ and $v$ in $M(\Omega)$ are Teichmüller equivalent, denoted by $\mu \sim v$, if there are two quasiconformal maps $f^{\mu}$ and $f^{v}$ induced by them respectively and a conformal map $c: f^{\mu}(\Omega) \longrightarrow f^{v}(\Omega)$ such that $\left(f^{v}\right)^{-1} \circ c \circ f^{\mu}$ is homotopic to the identity on $\Omega$ modulo the boundary $\partial \Omega$ of $\Omega$. The boundary can be considered as the ideal boundary as well as the topological boundary of $\Omega$ [2]. If we denote by $[\mu]$ the equivalence class containing $\mu$ for every $\mu \in M(\Omega)$, then

$$
T(\Omega)=M(\Omega) / \sim=\{[\mu] \mid \mu \in M(\Omega)\} .
$$

For more details on Teichmüller spaces, see, for example, [4], [5], [6] and [7].

Received by the editors November 8, 2000.

2000 Mathematics Subject Classification. Primary 30F60; Secondary 32G15.

Key words and phrases. Quasiconformal mapping, Teichmüller space.

This work was supported by the NSF Grant 19871084 and 19901032 of China and the China Postdoctoral Science Foundation. 
Let $\zeta$ be a point in the boundary $\partial \Omega$ and let $\mu \in M(\Omega)$. Denote

$$
h_{\zeta}^{*}(\mu)=\inf \left\{\left\|\left.\mu\right|_{U}\right\|_{\infty} \mid U \text { is an open disk in } \mathbf{C} \text { containing } \zeta\right\}
$$

and

$$
h_{\zeta}([\mu])=\inf \left\{h_{\zeta}^{*}(v) \mid v \in[\mu]\right\} .
$$

Then the local boundary dilatations at $\zeta$ of $\mu \in M(\Omega)$ and $\tau=[\mu] \in T(\Omega)$ are defined as

$$
H_{\zeta}^{*}(\mu)=\frac{1+h_{\zeta}^{*}(\mu)}{1-h_{\zeta}^{*}(\mu)} \quad \text { and } \quad H_{\zeta}([\mu])=\frac{1+h_{\zeta}([\mu])}{1-h_{\zeta}([\mu])}
$$

respectively [5]. Note that these definitions are somewhat different from the usual ones [9]. They depend on the topological boundary of $\Omega$.

The following problem was proposed by F. Gardiner and N. Lakic in [5] as an open problem.

Problem. Let $\zeta$ be a boundary point of a plane domain $\Omega$, and let $\tau \in T(\Omega)$. Is there a locally extremal Beltrami coefficient $\mu$ representing the class $\tau$ at the point $\zeta$ ? That is, can we find a Beltrami coefficient $\mu \in M(\Omega)$ such that $\tau=[\mu]$ and $h_{\zeta}^{*}(\mu)=h_{\zeta}(\tau)$ ?

In this paper, we give an affirmative answer to this problem in the case of $\Omega$ that is the unit disk $\Delta$ by studying the gluing of quasiconformal maps. Our main result is the following.

Theorem 1. Let $\zeta$ be a boundary point of the unit disk $\Delta$ and let $\tau \in T(\Delta)$. Then there is a Beltrami coefficient $\mu \in M(\Delta)$ such that $[\mu]=\tau$ and $h_{\zeta}(\tau)=h_{\zeta}^{*}(\mu)$.

The method used here can also be used to deal with some more general cases. However, there are some difficulties in solving the problem of F. Gardiner and N. Lakic completely.

\section{GLUing OF QUASICONFORMAL MAPS}

In order to prove Theorem 1, we need to glue two quasiconformal maps together to obtain a new quasiconformal map whose maximal dilatation can be controlled properly. The method used here is similar to that in [1]. The key tool is the following main inequality of Reich and Strebl [8].

Theorem A. Let $f$ and $g$ be quasiconformal homeomorphisms between Riemann surfaces $R$ and $R^{\prime}$ which are homotopic modulo the boundary. Then for every integrable holomorphic quadratic differential $\varphi=\varphi(z) d z^{2}$, we have

$$
\|\varphi\| \leq \iint_{\Delta}|\varphi(z)| \frac{\left|1-\mu_{f}(z) \frac{\varphi}{|\varphi|}\right|^{2}}{1-\left|\mu_{f}(z)\right|^{2}} D_{g^{-1}}(f(z)) d x d y
$$

where $D_{g^{-1}}(w)=\frac{1+\left|\mu_{g^{-1}}(z)\right|}{1-\left|\mu_{g^{-1}}(z)\right|}$ is the dilatation of $g^{-1}$ at $z$ and $\mu_{f}, \mu_{g}$ and $\mu_{g^{-1}}$ are the Beltrami coefficients of the quasiconformal homeomorphisms $f, g$ and $g^{-1}$ respectively.

To make our paper self-contained, we now introduce K. Strebel's definitions of local boundary dilatations and compare them with those given in section 1 .

From the definition of Teichmüller space, it is easily seen that for every $\mu \in$ $M(\Delta)$ there exists one and only one normalized (i.e. keep $i, 1$, and $-i$ fixed) 
quasiconformal map $f_{\mu}$ of $\Delta$ onto itself with complex dilatation $\mu$ and two Beltrami coefficients $\mu$ and $\nu$ in $M(\Delta)$ are Teichmüller equivalent if and only if $\left.f_{\mu}\right|_{\partial} \Delta=$ $\left.f_{\nu}\right|_{\partial} \Delta$. So the Teichmüller space of $\Delta$ (i.e. the universal Teichmüller space) can also be defined as the space of all normalized quasisymmetric maps of $\partial \Delta$ onto itself:

$$
T(\Delta)=:\{h: \partial \Delta \rightarrow \partial \Delta \mid h \text { is a normalized quasisymmetric map of } \partial \Delta\} .
$$

Let $h \in T(\Delta)$. The local boundary dilatation at $\zeta \in \partial \Delta$ and the boundary dilatation of $h$ are defined as

$$
\begin{aligned}
& H_{\zeta}(h)=: \inf \{K(f) \mid f \text { is a q.c. extension of } h \\
& \text { to a half neigborhood } U(\zeta) \subset \Delta \text { of } \zeta\}
\end{aligned}
$$

and

$$
\begin{aligned}
H(h)=: \inf \{K(f) \mid & f \text { is a q.c. extension of } h \\
& \text { to an annulus }(r<|z|<1) \text { and } 0 \leq r<1\}
\end{aligned}
$$

respectively [9]. It is proved by R. Fehlmann [3] that if $h$ is a self-homeomorphism of the unit circle $\partial \Delta$ and $H_{\zeta}(h)<\infty$ for every $\zeta \in \partial \Delta$, then $h$ is a quasisymmetric map of $\partial \Delta$ onto itself and

$$
H(h)=\max _{\zeta \in \partial \Delta} H_{\zeta}(h)
$$

It is clear that

$$
H_{\zeta}([\mu])=H_{\zeta}\left(\left.f_{\mu}\right|_{\partial \Delta}\right) \text { and } H_{\zeta}^{*}(\mu)=H_{\zeta}^{*}\left(f_{\mu}\right),
$$

where

$$
H_{\zeta}{ }^{*}\left(f_{\mu}\right)=: \inf \left\{K\left(\left.f_{\mu}\right|_{U(\zeta)}\right) \mid U(\zeta) \subset \Delta \text { is a half neigborhood of } \zeta\right\}
$$

is the local boundary dilatation at $\zeta$ of $f_{\mu}$. From now on, we will not distinguish these definitions from those given in section 1.

Notation. $\Delta(\zeta, r)=: \Delta \cap(|z-\zeta|<r)$ and $\Delta\left(\zeta ; r_{1}, r_{2}\right)=: \Delta \cap\left(r_{1}<|z-\zeta|<r_{2}\right)$.

Theorem 2. Let $f$ be a quasiconformal map of the unit disk $\Delta$ onto itself and let $\zeta \in \partial \Delta$ be a boundary point of $\Delta$. Let $g$ be a quasiconformal map of $\Delta\left(\zeta, r_{0}\right)$ into $\Delta$ with $\left.g\right|_{\partial \Delta \cap\left(|z-\zeta|<r_{0}\right)}=\left.f\right|_{\partial \Delta \cap\left(|z-\zeta|<r_{0}\right)}$ for some positive number $0<r_{0}<1$. Then for every $\epsilon>0$ there exist a positive number $r<r_{0}$ and a quasiconformal map $F$ of $\Delta$ onto itself with $\left.F\right|_{\partial \Delta}=\left.f\right|_{\partial \Delta}$ such that

(1) $F=f$ on $\Delta \backslash \Delta\left(\zeta, r_{0}\right)$;

(2) $F=g$ on $\Delta(\zeta, r)$;

(3) $K\left(\left.F\right|_{\Delta\left(\zeta ; r, r_{0}\right)}\right) \leq \max \left\{K\left(\left.f\right|_{\Delta\left(\zeta, r_{0}\right)}\right), K(g)\right\}+\epsilon$.

Proof. By the assumption of $g$, there is a positive number $\tilde{r}<r_{0}$ such that

$$
g(\Delta \cap|z-\zeta|=\tilde{r}) \subset f\left(\Delta\left(\zeta, r_{0}\right)\right) .
$$

For every positive number $r<\tilde{r}$, let

$$
h_{r}= \begin{cases}f & \text { on }\left(|z-\zeta|=r_{0}\right) \cap \Delta, \\ f=g & \text { on } \partial \Delta \cap\left(r \leq|z-\zeta| \leq r_{0}\right), \\ g & \text { on }(|z-\zeta|=r) \cap \Delta .\end{cases}
$$


It is clear that $h_{r}$ is a homeomorphism between two Jordan curves. Applying the well-known result of Fehlmann [3] mentioned above to the pullback of $h_{r}$ to the unit circle, we conclude that $h_{r}$ can be extended to $\Delta\left(\zeta ; r, r_{0}\right)$ quasiconformally and

$$
H\left(h_{r}\right) \leq \max \left\{K\left(\left.f\right|_{\Delta\left(\zeta, r_{0}\right)}\right), K(g)\right\} .
$$

Let $f_{r}$ be an extremal quasiconformal extension of $h_{r}$. If

$$
K\left(f_{r}\right) \leq \max \left\{K\left(\left.f\right|_{\Delta\left(\zeta, r_{0}\right)}\right), K(g)\right\}+\epsilon
$$

for some $r$, then

$$
F_{r}= \begin{cases}f & \text { on } \Delta \backslash \Delta\left(\zeta, r_{0}\right), \\ f_{r} & \text { on } \Delta\left(\zeta ; r, r_{0}\right), \\ g & \text { on } \Delta(\zeta, r)\end{cases}
$$

is the required quasiconformal map.

Now we assume that $K\left(f_{r}\right)>\max \left\{K\left(\left.f\right|_{\Delta\left(\zeta, r_{0}\right)}\right), K(g)\right\}+\epsilon$ holds for all positive numbers $r<\tilde{r}$. Then $K\left(f_{r}\right)>H\left(h_{r}\right)$ and consequently by the frame mapping theorem of Strebel [9] (also see, for example, [4], 5] and 7]), $f_{r}$ is a Teichmüller map with Beltrami coefficient $\mu_{r}=k_{r} \overline{\varphi_{r}} /\left|\varphi_{r}\right|\left(0<k_{r}<1\right)$, where $\varphi_{r}=\varphi_{r}(z) d z^{2}$ is the associated holomorphic quadratic differential with $L^{1}$-norm

$$
\left\|\varphi_{r}\right\|=\iint_{\Delta}\left|\varphi_{r}(z)\right| d x d y=1
$$

We claim that

Claim. $\varphi_{r}$ converges to 0 as $r \rightarrow 0$ uniformly on any compact subset of $\Delta\left(\zeta, r_{0}\right)$.

Assume, by contradiction, that there is a sequence $\left\{r_{n}\right\}$ of positive numbers with $r_{n+1}<r_{n}$ and $\lim _{n \rightarrow \infty} r_{n}=0$ such that $\varphi_{r_{n}} \rightarrow \varphi_{0} \not \equiv 0$ as $n \rightarrow \infty$, where $\varphi_{r_{n}}$ are the associated quadratic differentials of the Teichmüller maps $f_{r_{n}}$.

As the dilatations of $f_{r_{n}}$ are uniformly bounded from above by the dilatation of $F_{r_{1}}$, we can choose a subsequence of $\left\{f_{r_{n}}\right\}$, also denoted by $\left\{f_{r_{n}}\right\}$, such that $k_{r_{n}} \rightarrow k_{0}\left(0 \leq k_{0}<1\right)$ as $n \rightarrow \infty$. Together with the assumption that $\varphi_{r_{n}} \rightarrow \varphi_{0}$ (as $n \rightarrow \infty)$, the Beltrami coefficients $\mu_{r_{n}}$ of $f_{r_{n}}$ converge to $\mu_{0}=k_{0} \overline{\varphi_{0}} /\left|\varphi_{0}\right|$ in $\Delta\left(\zeta, r_{0}\right)$.

It is clear that the dilatation of $F_{r_{n}}$ are also uniformly bounded from above by the dilatation of $F_{r_{1}}$. So we can extend all $F_{r_{n}}$ to a larger disk $\Delta_{R}=:(|z|<R)$ $(R>1)$ symmetrically. The images of $\Delta_{R}$ under maps $F_{r_{n}}$ are contained in a disk whose radius is determined by $R$ and the dilatation of $F_{r_{1}}$. Therefore, $\left\{F_{r_{n}}\right\}$ is normal in $\Delta_{R}$. Hence $\left\{F_{r_{n}}\right\}$ has a subsequence, also denoted by $\left\{F_{r_{n}}\right\}$, which converges uniformly on any compact subset of $\Delta_{R}$ to a quasiconformal map $F_{0}$ defined in $\Delta_{R}$. In particular, $\left\{F_{r_{n}}\right\}$ converges to $F_{0}$ uniformly on $\bar{\Delta}$ and thus the restriction of $F_{0}$ to $\Delta$ is a quasiconformal map of $\Delta$ onto itself with the boundary values $\left.f\right|_{\partial \Delta}$.

From the definition of $F_{r_{n}}$ and the convergence of the Beltrami coefficients of $f_{r_{n}}$, we deduce by Theorem 4.5 of [6] that the Beltrami coefficients of $F_{r_{n}}$ converge to the Beltrami coefficient of $F_{0}$. The restriction of the Beltrami coefficient $\mu_{F_{0}}$ to $\Delta\left(\zeta, r_{0}\right)$ is equal to $\mu_{0}=k_{0} \overline{\varphi_{0}} /\left|\varphi_{0}\right|$. Thus $\left.F_{0}\right|_{\Delta\left(\zeta, r_{0}\right)}$ is an extremal Teichmüller map. On the other hand, the assumption that $K\left(f_{r}\right)>\max \left\{K\left(\left.f\right|_{\Delta\left(\zeta, r_{0}\right)}\right), K(g)\right\}+\epsilon$ holds for all positive numbers $r<\tilde{r}$ implies $K\left(\left.F_{0}\right|_{\Delta\left(\zeta, r_{0}\right)}\right) \geq K\left(\left.f\right|_{\Delta\left(\zeta, r_{0}\right)}+\epsilon\right.$. This gives a contradiction since $\left.f\right|_{\Delta\left(\zeta, r_{0}\right)}$ has the same boundary values with $\left.F_{0}\right|_{\Delta\left(\zeta, r_{0}\right)}$. Therefore, the proof of our claim is completed. 
Let $r^{\prime}<\tilde{r}$ be a fixed positive number. By the definition of $h_{r^{\prime}}$ and Fehlmann's result mentioned above,

$$
H\left(h_{r^{\prime}}\right) \leq \max \left\{K\left(\left.f\right|_{\Delta\left(\zeta, r_{0}\right)}\right), K(g)\right\} .
$$

So there are a compact subdomain $E$ of $\Delta\left(\zeta ; r^{\prime}, r_{0}\right)$ and a quasiconformal map $\tilde{h}_{r^{\prime}}$ defined on $\Delta\left(\zeta ; r^{\prime}, r_{0}\right) \backslash E$ with the boundary values $h_{r^{\prime}}$ such that

$$
K\left(\tilde{h}_{r^{\prime}}\right)<\max \left\{K\left(\left.f\right|_{\Delta\left(\zeta, r_{0}\right)}\right), K(g)\right\}+\frac{\epsilon}{2} .
$$

Moreover, $\tilde{h}_{r^{\prime}}$ can be extended to a quasiconformal map in the whole domain $\Delta\left(\zeta ; r^{\prime}, r_{0}\right)$. We denote also by $\tilde{h}_{r^{\prime}}$ one of its quasiconformal extensions and let

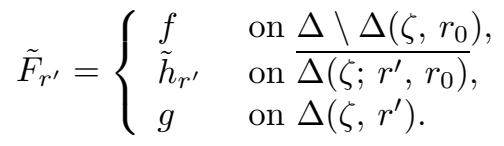

For any positive number $r<r^{\prime}, f_{r}$ is homotopic to the restriction of $\tilde{F}_{r^{\prime}}$ to $\Delta\left(\zeta ; r, r_{0}\right)$ modulo the boundary. By Theorem A, we have

$$
\begin{aligned}
1=\left\|\varphi_{r}\right\| & \leq \iint_{\Delta\left(\zeta ; r, r_{0}\right)}\left|\varphi_{r}(z)\right| \frac{\mid 1-\mu_{r}(z) \frac{\varphi_{r}(z)}{\left|\varphi_{r}(z)\right|^{2}}}{1-\left|\mu_{r}(z)\right|^{2}} D_{\tilde{F}_{r^{\prime}}^{-1}}\left(f_{r}(z)\right) d x d y \\
& =\iint_{\Delta\left(\zeta ; r, r_{0}\right)} \frac{\left|\varphi_{r}(z)\right|}{K\left(f_{r}\right)} D_{\tilde{F}_{r^{\prime}}^{-1}}\left(f_{r}(z)\right) d x d y .
\end{aligned}
$$

Thus,

$$
\begin{aligned}
K\left[f_{r}\right] & \leq \iint_{\Delta\left(\zeta ; r, r_{0}\right)}\left|\varphi_{r}(z)\right| D_{\tilde{F}_{r^{\prime}}^{-1}}\left(f_{r}(z)\right) d x d y \\
& =\iint_{f_{r}^{-1} \circ \tilde{F}_{r^{\prime}}(E)}\left|\varphi_{r}(z)\right| D_{\tilde{F}_{r^{\prime}}^{-1}}\left(f_{r}(z)\right) d x d y \\
& +\iint_{\Delta\left(\zeta ; r, r_{0}\right) \backslash f_{r}-1 \circ \tilde{F}_{r^{\prime}}(E)}\left|\varphi_{r}(z)\right| D_{\tilde{F}_{r^{\prime}}^{-1}}\left(f_{r}(z)\right) d x d y .
\end{aligned}
$$

As $K\left(f_{r}\right) \leq K\left(\tilde{F}_{r^{\prime}}\right)$, all $f_{r}^{-1} \circ \tilde{F}_{r^{\prime}}(E)\left(0<r<r^{\prime}\right)$ are contained in a compact subset of $\Delta\left(\zeta, r_{0}\right)$. By the degenerating property of $\left\{\varphi_{r}\right\}$,

$$
\iint_{f_{r}-1 \circ \tilde{F}_{r^{\prime}}(E)}\left|\varphi_{r}(z)\right| D_{\tilde{F}_{r^{\prime}}^{-1}}\left(f_{r}(z)\right) d x d y \leq \frac{\epsilon}{2}
$$

holds for all sufficiently small numbers $r$. By the definition of $\tilde{F}_{r^{\prime}}$ and (1),

$$
\iint_{\Delta\left(\zeta ; r, r_{0}\right) \backslash f_{r}-1 \circ \tilde{F}_{r^{\prime}}(E)}\left|\varphi_{r}(z)\right| D_{\tilde{F}_{r^{\prime}}^{-1}}\left(f_{r}(z)\right) d x d y \leq \max \left\{K\left(\left.f\right|_{\Delta\left(\zeta, r_{0}\right)}\right), K(g)\right\}+\frac{\epsilon}{2}
$$

and consequently,

$$
K\left(f_{r}\right) \leq \max \left\{K\left(\left.f\right|_{\Delta\left(\zeta, r_{0}\right)}\right), K(g)\right\}+\epsilon,
$$

which completes the proof of Theorem 2. 


\section{Existence of LOCALly extremal Beltrami COEFFicients}

Because of the preparation in section 2, we can now show the existence of locally extremal Beltrami coefficients.

From the definition of $h_{\zeta}([\mu])$, it is clear that there is a sequence $\left\{\mu_{n}\right\}$ of Beltrami coefficients, which are Teichmüller equivalent to $\mu$, such that

$$
\lim _{n \rightarrow \infty} h_{\zeta}^{*}\left(\mu_{n}\right)=h_{\zeta}([\mu])
$$

Without loss of generality, we can assume

$$
h_{\zeta}^{*}\left(\mu_{1}\right)>h_{\zeta}^{*}\left(\mu_{2}\right)>h_{\zeta}^{*}\left(\mu_{3}\right)>\cdots>h_{\zeta}^{*}\left(\mu_{k}\right)>\cdots .
$$

By the definition of $h_{\zeta}^{*}\left(\mu_{n}\right)$ and (3), there are two sequences $\left\{r_{k}\right\}$ and $\left\{\epsilon_{k}\right\}$ of positive numbers with $r_{k+1}<r_{k}$ and $\lim _{k \rightarrow \infty} r_{k}=\lim _{k \rightarrow \infty} \epsilon_{k}=0$ such that

$$
\left\|\left.\mu_{k+1}\right|_{\Delta\left(\zeta, r_{k+1}\right)}\right\|_{\infty}<\left\|\left.\mu_{k}\right|_{\Delta\left(\zeta, r_{k}\right)}\right\|_{\infty}-\epsilon_{k}<h_{\zeta}^{*}\left(\mu_{k}\right) \leq\left\|\left.\mu_{k}\right|_{\Delta\left(\zeta, r_{k}\right)}\right\|_{\infty}
$$

holds for all positive integers $k$.

Let $f_{k}$ be the normalized quasiconformal map of the unit disk onto itself with Beltrami coefficient $\mu_{k}$. Then $\left.f_{k}\right|_{\partial \Delta}=\left.f_{l}\right|_{\partial \Delta}$ for all positive integers $k$ and $l$. Applying Theorem 2 to $f_{1}$ and $f_{2}$, we get a positive number $r_{1}^{\prime}<r_{2}$ and a quasiconformal map $g_{1}$ of $\Delta$ onto itself such that

$$
\begin{aligned}
& \left.g_{1}\right|_{\partial \Delta}=\left.f_{1}\right|_{\partial \Delta}, \\
& g_{1}=f_{1} \text { on } \Delta \backslash \Delta\left(\zeta, r_{1}\right), \\
& g_{1}=f_{2} \text { on } \Delta\left(\zeta, r_{1}^{\prime}\right), \\
& \left\|\left.\mu_{g_{1}}\right|_{\Delta\left(\zeta ; r_{1}^{\prime}, r_{1}\right)}\right\|_{\infty}<\left\|\left.\mu_{1}\right|_{\Delta\left(\zeta, r_{1}\right)}\right\|_{\infty}+1 .
\end{aligned}
$$

Let $r_{k_{1}}=r_{2}$ and let $k_{2}$ be the least positive integer with $r_{k_{2}}<r_{1}^{\prime}$. Applying Theorem 2 to $g_{1}$ and $f_{k_{2}}$, we get a positive number $r_{2}^{\prime}<r_{k_{2}}$ and a quasiconformal map $g_{2}$ of $\Delta$ onto itself such that

$$
\begin{aligned}
& \left.g_{2}\right|_{\partial \Delta}=\left.f_{1}\right|_{\partial \Delta}, \\
& g_{2}=g_{1} \text { on } \Delta \backslash \Delta\left(\zeta, r_{1}^{\prime}\right), \\
& g_{1}=f_{k_{2}} \text { on } \Delta\left(\zeta, r_{2}^{\prime}\right), \\
& \left\|\left.\mu_{g_{2}}\right|_{\Delta\left(\zeta ; r_{2}^{\prime}, r_{1}^{\prime}\right)}\right\|_{\infty}<\left\|\left.\mu_{k_{1}}\right|_{\Delta\left(\zeta, r_{k_{1}}\right)}\right\|_{\infty}+\frac{1}{2} .
\end{aligned}
$$

By induction, we obtain two sequences $\left\{r_{k_{n}}\right\}$ and $\left\{r_{n}^{\prime}\right\}$ of positive numbers and a sequence $\left\{g_{n}\right\}$ of quasiconformal maps of $\Delta$ onto itself with the following properties:

$$
\begin{aligned}
& r_{n}^{\prime}<r_{k_{n}}<r_{n-1}^{\prime}, \\
& \left.g_{n}\right|_{\partial \Delta}=\left.f_{1}\right|_{\partial \Delta}, \\
& g_{n}=g_{n-1} \text { on } \Delta \backslash \Delta\left(\zeta, r_{n-1}^{\prime}\right), \\
& g_{n}=f_{k_{n}} \text { on } \Delta\left(\zeta, r_{n}^{\prime}\right), \\
& \left\|\left.\mu_{g_{n}}\right|_{\Delta\left(\zeta ; r_{n}^{\prime}, r_{n-1}^{\prime}\right)}\right\|_{\infty}<\left\|\left.\mu_{k_{n-1}}\right|_{\Delta\left(\zeta, r_{k_{n-1}}\right)}\right\|_{\infty}+\frac{1}{n},
\end{aligned}
$$

By the same method used in section 2, $\left\{g_{n}\right\}$ is normal in a disk that properly contains the closed unit disk. So there is a subsequence, also denoted by $\left\{g_{n}\right\}$, converging to a quasiconformal map $g_{0}$ uniformly on $\bar{\Delta}$. Thus $\left.g_{0}\right|_{\partial \Delta}=\left.f_{1}\right|_{\partial \Delta}$ and consequently, $\mu_{\left.g_{0}\right|_{\Delta}}$ is Teichmüller equivalent to $\mu$. From the definition of $g_{n}$, it is 
obvious that

$$
\mu_{g_{0}}=\lim _{n \rightarrow \infty} \mu_{g_{n}},=\left\{\begin{array}{ccc}
\mu_{f_{1}} & \text { on } \Delta \backslash \Delta\left(\zeta, r_{1}\right), \\
\mu_{g_{1}} & \text { on } \Delta\left(\zeta ; r_{1}^{\prime}, r_{1}\right), \\
\mu_{g_{2}} & \text { on } \Delta\left(\zeta ; r_{2}^{\prime}, r_{1}^{\prime}\right), \\
\cdots & \cdots & \\
\mu_{g_{n}} & \text { on } & \Delta\left(\zeta ; r_{n}^{\prime}, r_{n-1}^{\prime}\right), \\
\cdots & \cdots &
\end{array}\right.
$$

Together with (4) and (5), we have

$$
\left\|\left.\mu_{g_{0}}\right|_{\Delta\left(\zeta, r_{n-1}^{\prime}\right)}\right\|_{\infty} \leq\left\|\left.\mu_{k_{n-1}}\right|_{\Delta\left(\zeta, r_{k_{n-1}}\right)}\right\|_{\infty}+\frac{1}{n}
$$

for all positive integers $n$. From (2), (3) and (4), it is easily seen that

$$
\lim _{n \rightarrow \infty}\left\|\left.\mu_{k_{n-1}}\right|_{\Delta\left(\zeta, r_{k_{n-1}}\right)}\right\|_{\infty}=h_{\zeta}([\mu])
$$

Thus,

$$
h_{\zeta}^{*}\left(\mu_{g_{0}}\right)=\lim _{n \rightarrow \infty}\left\|\left.\mu_{g_{0}}\right|_{\Delta\left(\zeta, r_{n-1}^{\prime}\right)}\right\|_{\infty} \leq h_{\zeta}([\mu]) .
$$

On the other side, it is clear from the definition of $h_{\zeta}([\mu])$ that $h_{\zeta}^{*}\left(\mu_{g_{0}}\right) \geq h_{\zeta}([\mu])$. Therefore, $h_{\zeta}^{*}\left(\mu_{g_{0}}\right)=h_{\zeta}([\mu])$. This completes the proof of Theorem 1 .

\section{ACKNowledgement}

The authors would like to thank Professor Lo Yang and Professor Zhong Li for their constant support and encouragement. Thanks are also due to the referee for many suggestions and corrections.

\section{REFERENCES}

1. G. Cui, Geometrically finite rational maps with given combinatorics, Preprint, Nov., 1999.

2. C. Earle and C. McMullen, Quasiconformal isotopies, Holomorphic functions and Moduli, Vol. I, 143-154, edited by D. Drasin ect., Springer-Verlag, 1988. MR 89h:30028

3. R. Fehlman, Ueber extremale quasikonforme abbidungen, Comment. Math. Helv., 56(1981), 558-580. MR 83e:30024

4. F. Gardiner, Teichmuller theory and quadratic differentials, John Wiley and Sons, New York, 1987. MR 88m:32044

5. F. Gardiner and N. Lakic, Quasiconformal Teichmüller spaces, Math Surveys and Monographs, Vol 76, AMS., Providence, RI., 2000. MR 2001d:32016

6. O. Lehto, Univalent functions and Teichmüller spaces, GTM, Vol. 109, Springer-Verlag, New York, 1987. MR 88f:30073

7. Li, Z., Quasiconformal mappings with applications to Riemann surfaces (in Chinese), Science Press (1988).

8. Reich E. and Strebel K. Extremal quasiconformal mapping with given boundary values, Bull. Amer. Math. Soc. 79 (1973), 488-490.

9. Strebel, K., On the existence of extremal Teichmüller mappings, J. d'analyses Math., 30 (1976), 464-480. MR 55:12912

Institute of Mathematics, Academy of Mathematics and System Sciences, Chinese Academy of Sciences, Beijing 100080, China

E-mail address: gzcui@math08.math.ac.cn

Institute of Mathematics, Academy of Mathematics and System Sciences, Chinese Academy of Sciences, Beijing 100080, China

E-mail address: qiyi@math03.math.ac.cn

Current address: Department of Applied Mathematics, Beijing University of Aeronautics and Astronautics, Beijing 100083, China

E-mail address: yiqi1@yahoo.com 